\title{
Prime focus architectures for large space telescopes: reduce surfaces to save cost \\ J. B. Breckinridge ${ }^{* 1,2}$, and C. F. Lillie ${ }^{3}$ \\ 1. California Institute of Technology, Pasadena, CA. \\ 2. College of Optical Sciences, University of Arizona, Tucson, AZ \\ 3. Lillie Consulting LLC, Playa del Rey, CA.
}

\begin{abstract}
Conceptual architectures are now being developed to identify future directions for post JWST large space telescope systems to operate in the UV Optical and near IR regions of the spectrum. Here we show that the cost of optical surfaces within large aperture telescope/instrument systems can exceed $\$ 100 \mathrm{M} /$ reflection when expressed in terms of the aperture increase needed to over come internal absorption loss. We recommend a program in innovative optical design to minimize the number of surfaces by considering multiple functions for mirrors. An example is given using the Rowland circle imaging spectrometer systems for UV space science. With few exceptions, current space telescope architectures are based on systems optimized for ground-based astronomy. Both HST and JWST are classical "Cassegrain" telescopes derived from the ground-based tradition to co-locate the massive primary mirror and the instruments at the same end of the metrology structure. This requirement derives from the dual need to minimize observatory dome size \& cost in the presence of the Earth's 1-g gravitational field. Space telescopes, however function in the zero gravity of space and the 1$\mathrm{g}$ constraint is relieved to the advantage of astronomers. Here we suggest that a prime focus large aperture telescope system in space may have potentially have higher transmittance, better pointing, improved thermal and structural control, less internal polarization and broader wavelength coverage than Cassegrain telescopes. An example is given showing how UV astronomy telescopes use single optical elements for multiple functions and therefore have a minimum number of reflections.
\end{abstract}

Keywords: Telescopes, Prime focus, transmittance, LUVOIR, cost, schedule, ultraviolet,

\section{INTRODUCTION}

In this paper, we discuss the concept of prime focus instruments at a Large UV Optical Infrared (LUVOIR) space telescope ${ }^{1}$ and discuss the impact on cost, schedule and signal-to-noise ratio (SNR) of reducing the number of reflections. An instrument system at the prime focus of a space telescope may enables us to minimize scattered light, eliminate one structurally sensitive optical element (the secondary), minimize polarization aberrations, reduce thermal disturbances, maximize transmittance and reduce cost for observing extremely faint objects from the UV to the visible. And when used with an-off axis telescope, a prime focus instrument avoids the scattered and diffracted light caused by the secondary support structure.

Most large (3 to 6-meter) ground-based telescopes constructed before 1980 were designed to accommodate instruments at their prime focus and to switch (Mayall 4-m; Blanco 4-m, Subaru 8-m, SALT 10-m) between prime focus and Cassegrain focus. At first these instruments used photographic plates and were operated by astronomers riding in a "prime focus cage". When CCDs became available, however; the plates were replaced with CCD arrays, which allowed the astronomer to operate the instrument from the comfort of a (heated) control room. These first instruments had Fields of View (FOVs) of $\sim 14$ to 16 arc minutes, but in the last decade large CCD arrays and new for wide field correctors optical designs have enabled 2 to 3 degree diameter FOVs at the prime focus of these telescopes. Ground telescopes are constrained to operate at atmospheric temperature and within the Earth's atmosphere and gravitational field. For space telescopes, however these ground-based constraints are missing and a prime focus space telescope system may be less expensive to build with higher science yield than the classic configurations now used by HST and JWST.

In section 2 we discuss a notional large space telescope with a prime focus, present a conceptual architecture, explore image quality and exoplanet imaging issues, study the FOV, and identify four potential advantages to large space

\footnotetext{
*jbreckin@earthlink.net; phone 1 (626) 318-0339
} 
telescope prime focus. In section 3 we present a calculation of the cost to the Project for unnecessary mirrors in the telescope/instrument system. In section 4 we look at UV applications and section 5 is a summary \& conclusions.

\section{NOTIONAL LARGE SPACE TELESCOPE WITH PRIME FOCUS}

\section{Optical design}

Figure 1 is a sketch of a notional prime focus telescope system designed for wide-field and coronagraphic imaging by a 16-meter class telescope. Light passes from the right to the left to reflect from the primary mirror, segmented primary mirror. The box shown just to the right of the prime focus is the prime focus instrument assembly (PFIA) whose diameter is 3 to 4 meters and length a few meters to hold prime focus instruments such as UV spectrometers, imagers, coronagraphs and wide-field cameras. The focal plane is shared (like HST and JWST) with several instruments contained within the PFIA.

We select one example instrument, an imaging system, for discussion here. In our notional design, the prime focus is 24-m from the vertex of the primary. Light passes through a field lens and expands to fill the primary of an inverse Schwarzschild ${ }^{2}$. This reimaging system is mounted just beyond prime focus. The field lens relays an image of each segment of the primary onto the 0.6-meter diameter active segmented first reflecting surface of the Schwarzschild. Primary mirror wavefront errors (tilt, piston and surface) caused by fabrication and time-dependent thermal, dynamics and structural effects are compensated for at the $60-\mathrm{cm}$ diameter segmented active Schwarzschild primary mirror. Light reflects to the convex tertiary and then to the focal plane indicated by the circle or to a dispersive element in the case a spectrometer is chosen. The convex curvature on the tertiary combines with the concave powered mirrors 1 and 2 to provide a flat field at the focus. Adjusting the design of the Schwarzschild-relay controls the detector sampling frequency. For small fields of view such as those needed for exoplanet coronagraphy and high contrast imaging of stellar neighborhoods, the transparent field lens could be swung out of the way and replaced by a complex mask for unprecedented direct control of the unwanted radiation after the first reflection. There are no fold mirrors in the system, which will minimize polarization aberrations and their deleterious effect on the $\mathrm{PSF}^{4,5}$.

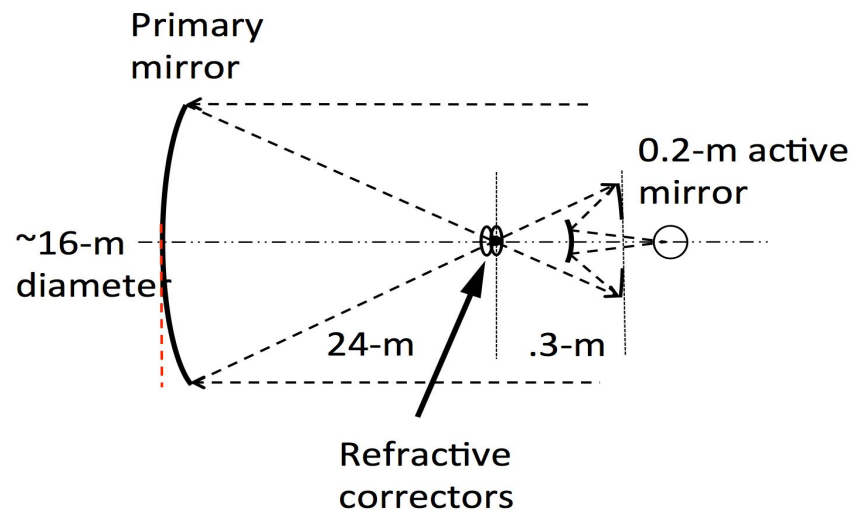

Figure 1: Shown is the schematic of a notional prime focus system. We selected a 16-m segmented diameter $\mathrm{F} / \#=1.5$, with a 24-m focal length and a reverse Schwarzschild relay with a $20-\mathrm{cm}$ segmented active mirror and a convex secondary. This system has a minification of 80 to be compared with the HST WF/PC2 value of $\sim 200$. Refractive correctors with internal baffles are used at the prime focus to increase the FOV for UV-Vis applications. There is a field stop at the prime focus. The circle on-axis at the right end of the optical system indicates the location of an instrument: spectrometer or coronagraph..

\section{Image quality \& exoplanet applications}

Breckinridge $^{4}$ and Chipman ${ }^{5}$ showed that polarization accumulated by wavefronts as they propagate through an optical system has an important role in image quality. The polarization aberrations: diattenuation and retardance change the shape and intensity of the system PSF, in addition to causing a faint but important broad "skirt" or tapered plateau of light which is centered around the base of the PSF where exoplanets hide within the diffraction pattern. The intensity of this light increases as the square ${ }^{5}$ of the number of uncompensated reflections in the system. Coronagraphs designed for 
the LUVOIR and HabEx optical systems, which will characterize terrestrial exoplanets need to minimize the number of reflecting surfaces and balance or mitigate the polarization aberrations.

\section{Wide field of View}

High quality wide field imaging array at the prime focus is achieved using a two to four element refractive corrector. C. G. Wynne ${ }^{6}$ designed prime focus field correctors for the Blanco 4-meter telescope at Cerro Tololo (parabolic) and correctors. Near diffraction-limited fields of view up to 40 arc minutes have been achieved at a 4-meter telescope $\mathrm{F} / \#=2.7^{7}$. The dominant aberrations are coma and astigmatism both of which affect the symmetry of the PSF. If we use the structural aberration coefficients developed by Gardner ${ }^{8}$ and Sasian ${ }^{9}$ used by Breckinridge ${ }^{10}$ we find that coma increases linearly with the product of diameter times field angle and that astigmatism increases linearly with diameter times the square of the field angle. At wide fields astigmatism will dominate. Scaling we find that a 12-meter should have diffraction-limited performance over a 5-arc minute FOV. The 12-m EST diffraction spot size of 8 milliarcseconds at $400 \mathrm{~nm}$ gives $>10^{+9}$ independent Nyquist sampled resolution elements across the 5-arc minute FOV object space. Additional research into innovative optical designs specifically focused on increasing this field of view may double the FOV.

Radiation damage to glasses was extensively studied by the Kepler project, which uses a Schmidt transparent lens corrector. Glass types with the correct dispersion and index properties that also survive long duration in space have been studied ${ }^{11}$, but additional work is needed.

\section{Prime focus potential advantages}

The potential advantages of prime focus space telescope that need further investigation are:

1. In Fig 1, we see that there are only three reflections from powered optical elements and no reflections from fold mirrors before the focal plane. The refractive correctors needed for wide field applications ${ }^{12}$ have low curvature. This will minimize reflection losses and minimize system Fresnel polarization aberrations to maximize system transmittance for imaging objects at the threshold of detection and UV spectroscopy.

2. For exoplanet coronagraphy, since the polarization aberrations ${ }^{13}$ are minimal, there will probably be insignificant difference between the aberrations in the two orthogonal polarizations and no need to polarizationdivide the pupil-image for optimal adaptive optics correction. This will avoid a 50\% loss in system transmittance.

3. In a classical Cassegrain system such as HST and a TMA such as JWST, the telescope optical path passes twice along the metering structure separating the primary and secondary. Length errors caused by thermal changes and structural dynamics of this metering structure are doubled in a Cassegrain configuration compared to a prime focus system.

4. Because the PFIA looks directly at the primary mirror, the prime focus requires less baffling than a system, which locates the instruments behind the primary. This saves mass and reduces system complexity to reduce cost and eliminate sources of unwanted radiation, which create background noise on the detector.

Further investigations are needed to explore possible other advantages of the prime focus such as: Free form optics - The diffraction limited FOV achievable with the inverse Schwarzschild, in the presence of the radiation-hard apochromatic prime-focus aspheric correctors will be better optimized using modern free-form optimization ${ }^{14}$. Polarization aberration balancing will be needed to optimize the coronagraph contrast. Telescope dynamics - Advantages of the two-body dynamics of the prime-focus (primary and instruments) compared to the three body (primary, secondary, instruments) dynamics if the instruments are behind the primary.

\section{COST OF MIRRORS}

\section{More optical surfaces than necessary}

Each reflection and every dielectric surface in a space telescope/instrument system has several technical performance and financial costs associated with it. These are: 
1. Absorption: The cost to the system performance (SNR) in terms of radiation lost by absorption and internal polarization $^{15}$ through the physical process of reflection of the electromagnetic wave. This results in increased integration time, reduced threshold brightness and less science during mission lifetime.

2. Mounts and fixtures: Cost and schedule to design, fabricate, test, integrate and align precision mechanical systems that provide stable support and accurate metrology for the optical elements within the system. This is required in the presence of spacecraft and mission thermal and dynamic disturbances.

3. Optical surfaces: Cost and schedule to design, fabricate, test mirror surfaces and their coatings.

4. Control of scattered light: Cost to design, fabricate, coat, test and align mechanical baffles to control unwanted radiation.

5. Polarization aberrations: Cost to mitigate or balance unwanted polarization aberrations ${ }^{16}$.

Clearly if we reduce the number of optical surfaces between the primary mirror and the science instrument focal plane, there is a very high probability that cost will be reduced. Assigning a specific cost to each of the items in the list above is not possible without a detailed design for the system. However we can take a different look at this problem and estimate costs.

The diameter of the telescope primary mirror determines the system angular resolution and the surface area encompassed by this diameter determines the amount of power, and thus SNR at the focal plane. If we constrain our argument to power at the focal plane, we obtain an estimate the of cost at the systems level based on the growth of the telescope aperture required to hold the focal plane flux constant (science SNR) in the presence of an increasing the number of mirror surfaces. The effective aperture of a telescope, $A_{e}$, for a system whose transmittance is $\tau$ is given by

$$
A_{e}=\tau A_{T}
$$

Eq. 4

where $A_{T}$ is the light collecting area of the telescope. The cost of a telescope increases with aperture and largeaperture space-telescope engineers ${ }^{4}$ believe that the cost $C$ increases as the 2.2 power of the aperture or

$$
C \propto A_{T}^{2.2}
$$

The entire optical system includes all mirrors and surfaces in both the instrument and the telescope. If we let $R_{i}$ be the reflectivity of the $i$ th mirror out of a total of $k$ mirrors and $S_{j}$ be the transmittance of the $j$ th surface out of a total of $l$ surfaces in the system, then the total intensity scalar transmittance $\tau$ of a telescope is given by

$$
\tau=\left(\prod_{i=1}^{i=k} R_{i}\right) \cdot\left(\prod_{j=1}^{j=l} S_{j}\right)
$$

For this discussion, we consider the losses caused by reflections from the highly reflecting broadband metal coatings required to have a high-transmittance telescope.

Equations 4 through 6 enable us to assign an average cost factor per optical reflection to a telescope optomechanical design. Table 1 shows the growth of telescope diameter and cost required to hold the focal plane flux constant in the presence of an increasing the number of mirror surfaces used by the optical designer as a function of the average reflectivity of the mirrors within the optical system, assuming that cost increases as the 2.2 power. The number of reflections shown varies from 4 to 20. Telescope diameter and cost is shown for 98,95 and 90 percent reflectivity for each mirror for the total number of optical system reflections between 4 and 20 .

Table 1 shows that if we assume our end-to-end optical system has 8 reflections then in order to overcome those power losses incurred by using 98\% reflectivity optics requires that we increase the diameter by $7 \%$ and the cost increases by $17 \%$. The LUVOIR telescope system concepts range from 12 to 20 -meter diameter aperture. We assume a baseline 16-meter aperture at a cost of $\$ 5 \mathrm{~B}$ and calculate a cost increase to restore the power absorbed by the 8 mirrors. This cost is $\$ 850$ million or about $\$ 100$ million per mirror.

The 98\% reflectivity per mirror we assumed in the above calculation is very optimistic. Mirrors are coated and then integrated into an optical system over a period of months. Even in a clean room mirror 
reflectivities typically degrade to about $96 \%$ or less before launch, to bring the cost, in terms of aperture up to well over $\$ 100$ million for each reflection.

It would seem prudent to expend some technology dollars today to investigate innovative designs for imaging and spectrometer and integral field unit systems to

1. Reduce reflections by having single optical elements perform multiple functions,

2. Develop innovative opto-mechanical packaging,

3. Compensate polarization aberrations,

4. Increase the reflectivity of highly reflecting thin films, and

5. Improve optical contamination control processes.

Table 1. Growth of telescope diameter and cost required to maintain the focal plane flux constant in the presence of an increasing the number of mirror surfaces used by the designer within the optical path. Column 1 shows mirrors reflectivities of 98,95 and 90 percent. Columns 2 through 5 give the diameter and cost factor increase needed for a 4, 8, 12, 16 and 20-meter aperture.

\begin{tabular}{|c|c|c|c|c|c|c|c|c|c|c|}
\hline $\begin{array}{l}\text { Number of } \\
\text { reflections }\end{array}$ & \multicolumn{2}{|c|}{4} & \multicolumn{2}{c|}{8} & \multicolumn{2}{c|}{12} & \multicolumn{2}{c|}{16} & \multicolumn{2}{c|}{20} \\
\hline \hline $\mathrm{R} \%$ & Dia. & Cost & Dia. & Cost & Dia. & Cost & Dia. & Cost & Dia. & Cost \\
\hline 98 & 1.03 & 1.08 & 1.07 & 1.17 & 1.12 & 1.28 & 1.16 & 1.40 & 1.21 & 1.53 \\
\hline 95 & 1.08 & 1.18 & 1.20 & 1.48 & 1.33 & 1.86 & 1.47 & 2.33 & 1.63 & 2.92 \\
\hline 90 & 1.17 & 1.42 & 1.45 & 2.25 & 1.79 & 3.58 & 1.20 & 5.69 & 2.72 & 9.04 \\
\hline
\end{tabular}

\section{ULTRAVIOLET SPECTROPHOTOMETRY}

Mirror reflectivities in the mid UV (90 to $150 \mathrm{~nm}$ ) are low (Keski-Kuha et al 1999). The prime focus of a telescope is an ideal location for an ultraviolet spectrometer, as demonstrated by several sounding rocket experiments by Lillie \& Lawrence ${ }^{17}$ and others ${ }^{18}, 19$ and the very successful Hopkins Ultraviolet Telescope (HUT) that was flown as a Spacelab experiment on the space shuttle in 1990 and 199520,21

In order to achieve the high sensitivity needed to record spectra of faint ultraviolet objects, optical designs that minimize the number of reflections are required to compensate for the low reflectivity of mirror coatings at wavelengths less than 110-nm. Clearly the minimum number of elements is two surfaces: one, a large aperture to collect and image radiation onto a focal plane stop at the entrance to a spectrometer and the other a single element that combines the functions of dispersion, wavefront correction and focusing.

\section{The Rowland circle spectrograph}

\section{The Rowland circle spectrograph}

Here we provide an example of how these functions are combined to provide the science community with the highest possible transmittance system for UV spectroscopy. Both are based on the principle of the Rowland circle. Figure 1 shows the simplest possible broad-band spectrometer. The entrance slit to the spectrograph extends normal to the sheet in Fig 1. It is located at the image plane of an imaging system. The light that converges to the mage plane where the slit is located is traveling right to left in this drawing. The slit is located near the center of curvature of the spherical diffraction grating. Light diverges after this slit to fill the spherical concave diffraction grating, which is shown to have radius $r$. The reflected light is quasimonochromatic and the spectrum of the source admitted to the spectrograph by the entrance slit dispersed onto a curved focal plane of radius $\mathrm{r} / 2$ shown in Fig 1 between $\lambda_{1}$ to $\lambda_{2}$. 


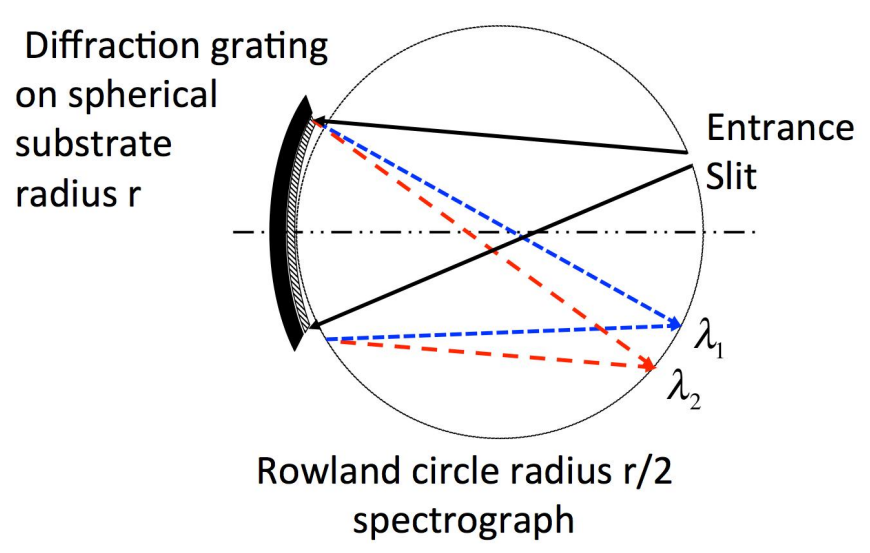

Figure 1. Sketch of the Rowland circle spectrograph. Light, travelling right to left enters the slit in the upper right and fills the concave grating of radius $r$. Light reflects from the grating and is focussed by the optical power onto the curved grating substrate onto a focal surface at radius of $\mathrm{r} / 2$ as shown in this figure. The slit length is extended normal to the page and the $r / 2$ is the surface corrected for the sagittal image, which is also normal to the page.

While the classical Rowland circle spectrometer with a concave spherical mirror provides good spatial resolution on a curved surface in the (x-y) plane of the Rowland circle, the slit images suffer from a large amount of astigmatism in the out of plane (y-z) direction, leading to tall, curved slit images. This focal plane curvature can be adequately corrected 222324 by curving the grating grooves to produce a flat field, with some loss in resolution.

Figure 2, below shows how this spectrometer is used in a UV spectrometer telescope system.

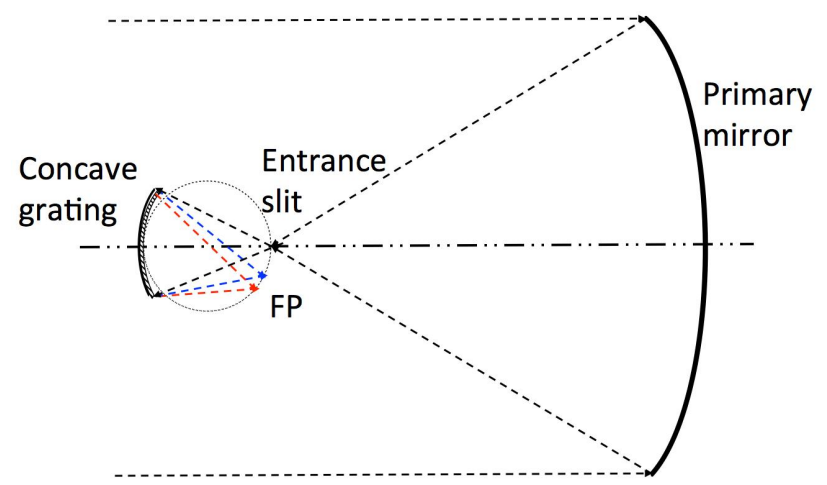

Figure 2. Optical configuration for a classic UV spectrometer. UV radiation enters the system from left to right, reflects from the primary mirror and object space is imaged onto the entrance slit of the spherical concave grating spectrograph. I the radius of curvature of the grating is $r$, then the radius of curvature of the aberration corrected field is $\mathrm{r} / 2$. This is a two-mirror prime focus telescope system.

\section{Prime focus UV flight systems}

Two successful flight instruments that employ this design approach for UV spectrometers are discussed. The first is a mechanically robust system designed for rocket launch ${ }^{25}$. In this case the focus of a 40$\mathrm{cm}$ diameter parabolic primary mirror of a $40-\mathrm{cm}$ telescope is co-incident with the focus of an 11 -cm concave, holographically ruled diffraction grating. This creates a Rowland circle spectrometer in which both the entrance aperture and the array detector [a micro-channel plate (MCP)] are located on a circle tangent to the grating surface, with a radius of $1 / 2$ the grating's radius of curvature. The primary mirror is a $40-\mathrm{cm} \mathrm{F} / \#=2.5$, Cervit MgF2Al coated primary that feeds an 11-cm diameter F/\# 1.25, 1200 line/mm concave LiAl coated holographic grating in a housing with a LiF window, evacuated to $10^{-6}$ torr with a vac-ion pump. The micro-channel plate was coated with CsI to convert the high-energy photons to electrons before the gain. The MCP had a 1024 coded anode array, 
which gave measured $1.5 \mathrm{~nm}$ resolution from 108 to $181 \mathrm{~nm}$. It was reported ${ }^{19}$ that the instrument provided 0.75 $\mathrm{nm}$ spectral resolution from 40 to $126 \mathrm{~nm}$ in the laboratory.

The second example we will discuss is the Hopkins Ultraviolet Telescope (HUT) with its prime focus spectrograph (Davidson, et al 1981) ${ }^{20}$, The HUT was developed at Johns Hopkins as one of three instruments mounted on the Spacelab Instrument Pointing Systems (IPS) a three-axis gimbaled mounting platform used on an exposed pallet in the payload bay of the Space Shuttle. The HUT optical system had a 0.9-m F\#/2 iridium coated parabolic primaryand a near-normal incidence Rowland-circle spectrograph. The 20-cm F\#/2 spherical concave holographic grating had $600 \mathrm{l} / \mathrm{mm}$ and was overcoated with osmium and was housed in a stainless steel container evacuated to 10-6 torr. . The detector located on the sphere shown in Fig 1 was a CsI coated MCP intensifier mounted to a 1024 channel diode array. The instrument gave $0.3 \mathrm{~nm}$ resolution in $1^{\text {st }}$ order from 85 to $185 \mathrm{~nm}$ and $0.15 \mathrm{~nm}$ resolution from 41.5 to $82.5 \mathrm{~nm}$ in $2^{\text {nd }}$ order. HUT observed 77 targets during the 10-day STS-35 Astro-1 mission in December 1990 and 275 targets during the 14-day STS-67 Astro-2.

In order to image a point in the entrance aperture to a point in the focal plane, the astigmatism and other aberrations of grating-based spectrometers can be reduced by using aspheric grating substrates with asymmetrically distributed grooves, produced either holographically or by sophisticated ruling engines. Examples of these approaches are described by Cotton ${ }^{26}$, who designed a single-element, hybrid RowlandCircle/Wadsworth imaging spectrograph with a toroidal grating; and by Fink ${ }^{27}$, et al 1996 who presented their calculations for aberration-corrected holographic gratings for 'two dimensional spectroscopy'. Clearly measurements across the bandwidth 50 to $150 \mathrm{~nm}$ will use prime focus systems.

\section{Large aperture UV spectrometer systems}

The next generation large aperture telescope optical systems with UV capability, such as the LUVOIR will face many new technical challenges including: 1 . Precision wavefront sensing and control (WF/SC), 2. Large area high reflectivity uniform thin films for the primary mirror, 3. Comprehensive contamination control and 4. Photon counting array detectors, in addition to innovative, UV capability-driven imaging spectrometers and spectropolarimeters.

Further development of this technology, perhaps with free-form optics, along with improved mirror coatings and high efficiency, low noise detector arrays will form the basis for the next generation of ultraviolet imaging spectrometers for future LUVOIR space telescopes.

Further development of this technology, perhaps with free-form optics, along with improved mirror coatings and high efficiency, low noise detector arrays will form the basis for the next generation of ultraviolet imaging spectrometers for future LUVOIR space telescopes.

\section{SUMMARY \& CONCLUSIONS}

In this paper we develop a cost metric for the number of reflections in a space astronomical

telescope/instrument system, and show that for a large telescope of the LUVOIR class, the "cost" of a single reflection approaches $\$ 100$ million. One way to reduce reflections is to eliminate the secondary optical element and use a prime focus system. We state that the advantages of a prime focus system are: possible reduced sensitivity of structural deformations caused by thermal and dynamics, improved telescope baffles, reduced polarization aberrations, and higher SNR because of increased transmittance.

As an example we examine the success that UV astrophysics has had using a simple two-element optical system: large primary and a Rowland circle spectrograph. By combining the functions of dispersion, wavefront correction and imaging into one optical element at prime focus the Rowland spectrograph has enabled far UV exploration of the universe. 


\section{REFERENCES}

[1] Dalcanton, J., et al, "From Cosmic Birth to Living Earths: The Future of UVOIR Astronomy," Association of Universities for Research in Astronomy (AURA) (2015)

[2] Wetherell, W. B. and Rimmer, M. P., "General analysis of aplanatic Cassegrain, Gregorian, and Schwarzschild telescopes", Appl. Opt. 11, 2817-2832 (1972)

[3] Budano, A., Flora, F. and Mezi, L. "Analytical design method for a modified Schwarzschild optics," Appl. Opt. 45, 4254-4262 (2006).

[4] Breckinridge, J. B., Lam, T. and Chipman, R., "Polarization aberrations in Astronomical Telescopes: The Point Spread Function," Pub. Astr. Soc. Pacific 127, 445-468 (2015)

[5] Chipman, R. A., Lam, Wai Sze T. and Breckinridge, J. "Polarization aberrations in astronomical telescopes," Proc SPIE 9613 doi:10.1117/12.2188921 (2015)

[6] Wynne, C. G., "Field correction for very large telescopes," Mon. Not. R. Astron. Soc 273, L45-L46 (1995)

[7] Wynne, C. G., "Wide field imaging," Mon. Not. R. Astron. Soc 236, Short communications 47-50 p. L45-L46 (1989)

[8] Gardner, I. C., "Applications of the algebraic aberration equations to optical design," Scientific papers for the

National Bureau of standards 22, US Gov’t printing office, pp 73-202 (1927)

[9] Sasian, J., "Introduction to aberrations in optical imaging systems," Cambridge University Press, ISBN 978-1-10700633-1 Ch 11 pp 147-162. (2013)

[10] Breckinridge, J. B. "Basic optics for the astronomical sciences," SPIE Press, Bellingham, WA; ISBN 978-0-81948366-9, Pages 84 - 99 (2012)

[11] Liu, H., et al. Effects of space environment factors on optical materials , Journal of spacecraft and rockets,

Volume: 42 Issue: 6 Pages: 1066-1069 (2005)

[12] Meinel, A. B., and Meinel, M. P., Two-stage optics: High acuity performance from low-acuity optical systems, Optical Engineering 31 2271-2281 (1992)

[13] Breckinridge, J. B., Lam, T. and Chipman, R., "Polarization aberrations in Astronomical Telescopes: The Point Spread Function," Pub. Astr. Soc. Pacific 127, 445-468 (2015)

[14] K. Fuerschbach, J. P. Rolland, and K. P. Thompson (2014) Theory of aberration fields for general optical systems with free-form optical surfaces, Optics Express 22, 26585-26606

[15] Breckinridge, J. B. and Chipman, R. A. "Telescope polarization and image quality: Lyot coronagraph performance," Proc. SPIE 9904-042 (2016)

[16] R. A. Chipman, Wai Sze T. Lam and J. Breckinridge "Polarization aberrations in astronomical telescopes," Proc SPIE 9613 doi:10.1117/12.2188 (2015)

[17] Lillie, C. F., and Lawrence, G. M., "A Rocket-Borne Faint Object Spectrograph with a Codacon Detector," Proc. of the SPIE 0172, Instrumentation in Astronomy III, 321 (1979)

[18] Lawrence, G. M. and Stone, E. J., “Ultraviolet spectrograph using microchannel plates,” Rev. Sci. Instrum. 46, 432 (1975)

[19] Davidson, A. F., Hartig, G. F., Fastie, W. G., "Ultraviolet spectrum of quasi-stellar object 3C273,” Nature 269, 203 - 206 (1977)

[20] Davidson, A. F., et al, “The John Hopkins Ultraviolet Telescope for Shuttle Astronomy.” Proc. SPIE 0265, Shuttle Pointing of Electro-Optical Experiments, 375 (1981)

[21] Dixon, W. V., et al, "The Hopkins Ultraviolet Telescope: The Final Archive," Publications Of The Astronomical Society Of The Pacific, 125:431-443, (2013)

${ }^{22}$ Grange, R., , “Aberration-reduced holographic spherical gratings for Rowland circle spectrographs,” Applied Optics Vol. 31, Issue 19, pp. 3744-3749, 1992

${ }^{23}$ Namioa, T. and Koike, M., “, Aspheric wave-front recording optics for holographic gratings,” Applied Optics Vol. 34, Issue 13, pp. 2180-2186, 1995

${ }^{24}$ Duban, M., "Theory and computation of three Cosmic Origins Spectrograph aspheric gratings recorded with a MDM," Appl. Opt. 38, 1096-1102. 1999

[25] Rocket system reference

[26] Cotton, D. M., Cook, T., and Chakrabarti, S., "Single-element imaging spectrograph,” Applied Optics 33, 1958 (1994)

[27] Fink, U., Rostalski, H. J., and Hirsch, H., "Applications of holographic gratings to two dimensional spectroscopy," Applied Optics 35, 1047 (1996)

[25[ Keski-Kuha, R. A. M., Larruquert, J. I., Gum, J. S., and Fleetwood, C. M., [Optical Coatings and Materials for Ultraviolet Space Applications], Ultraviolet Optical Space Astronomy Beyond HST, J.A. Morse, J.M. Shull and A. L. Kinney, eds., ASP Conference Series Vol. 161, pp. 406-419 (1999) 